\title{
Lesion homogeneity on diffusion-weighted imaging is a marker of outcome in acute ischemic stroke
}

\author{
Shady Mourad Boss, Ramez Reda Moustafa* (D), Magdy Ahmed Moustafa, Ahmed El Sadek, \\ Mohamed Mahmoud Mostafa and Hany Mohamed Aref
}

\begin{abstract}
Background and purpose: MRI Diffusion-weighted imaging (DWI) lesion volume, pattern, and location have all been used to predict outcome of acute ischemic stroke. We hypothesized that homogeneity within the DWI lesion may also be associated with outcome.

Methods: Brain MRI including DWI was performed for consecutive acute ischemic stroke patients within $48 \mathrm{~h}$ of onset. Patients were classified as having a homogenous or non-homogenous (heterogeneous) DWI lesion visually. NIHSS was recorded at baseline, 1 week and at 1 month. Modified Rankin Scale (mRS) score was assessed at 3 months.

Results: Fifteen patients were recruited to each group (homogenous and non-homogenous DWI lesion). There were no significant differences at baseline $(p>0.05)$. Patients with a homogenous DWI lesion had significantly higher mRS score at 3 months (worse outcome) than those with a non-homogenous (heterogeneous) DWI lesion (median, IQR 4, 2-4 vs. 1, 0-2 respectively, $p=0.001$ ). On repeated measures ANOVA, NIHSS was significantly worse at 1 week and 1 month in patients with a homogenous DWI lesion.
\end{abstract}

Conclusion: Lesion homogeneity on early MR DWI is a simple and reproducible visual assessment that may be a strong marker of outcome in acute ischemic stroke.

Keywords: Stroke, DWI, Lesion, Homogenous, Heterogeneous, Outcome, Predictor

\section{Introduction}

Predicting prognosis and outcome in acute ischemic stroke is important, particularly in identifying patients who may benefit from reperfusion therapies and those in which therapy would be futile or harmful. Structural MRI is a useful tool in predicting outcome and several MR $[1,2]$, and particularly diffusion-weighted imaging (DWI) characteristics [3-6], have been identified for this purpose. Lesion volume on DWI images is the most consistent marker of prognosis in most studies, but its use alone to exclude eligible patients has been challenged [7].

The DWI lesion is believed to reflect restricted water diffusion in the tissue which in turn approximates the infarct core and correlates strongly to final infarct

\footnotetext{
* Correspondence: ramezm@msn.com

Department of Neurology, Faculty of Medicine, Ain Shams University, 2nd Floor, Abbassia, Cairo, Egypt
}

volume $[8,9]$. Plenty of evidence suggests, however, that the DWI lesion may not be entirely composed of irreversibly damaged core and that it may contain "islands" of ischemic yet viable (salvageable) tissue [10-12]. Signal intensity on DWI potentially correlate in these varying degrees of ischemia and would be apparent on visual inspection. We thus hypothesized that acute ischemic stroke patients who have homogenous DWI lesions on MRI within 48 of stroke onset may have a worse outcome than those with non-homogenous (heterogeneous) DWI lesions.

\section{Methods}

This was a prospective case-control study. Consecutive acute ischemic stroke patients were prospectively recruited. Inclusion criteria were stroke onset within $48 \mathrm{~h}$, MRI brain with DWI was available, non-lacunar anterior circulation infarction on MRI, no significant pre-stroke 
disability (Modified Rankin Scale score $<2$ ) and age $>18$ years. Patients were excluded if they had significant concurrent electrolyte imbalance or uncontrolled general metabolic derangements.

\section{MRI}

Brain MRI was acquired using a $1.5 \mathrm{~T}$ machine (Signa Prospeed, GE, USA) including Fast Spin Echo T1W (TE/TR 9/520 ms), T2W (TE/TR 105/5000 ms), axial fluid-attenuated inversion recovery (FLAIR) (TE/TR $140 / 8000 \mathrm{~ms}$, inversion time $2 \mathrm{~s}$ ), axial single-shot multi-slice diffusion-weighted echo-planar imaging pulse sequence $($ DWI-EPI) $(b$ value $=1000$, TE/TR $112 / 6300 \mathrm{~ms})$ and EPI gradient echo T2* (TE/TR 15/540 ms), and timeof-flight MRA (TE/TR 6.9/36 ms).

\section{DWI homogeneity}

Visual assessment of DWI images was done by two examiners to classify lesions into homogenous and nonhomogenous (heterogeneous). A DWI lesion was considered homogenous if there was (1) no clearly discernable or very little differences in signal intensity in different parts of the lesion (featureless uniform hyperintensity); (2) loss of grey-white matter differentiation if lesion involved the cortex; (3) loss of structural details of the parenchyma within the lesion; and (4) no clear hemorrhagic transformation within the lesion (Fig. 1). Lesions were considered non-homogenous (heterogeneous) if they did not fulfil these criteria (i.e., showed variable degrees of hyperintensity in various parts of the lesion). Using these simple criteria, there was excellent inter-rater or intra- rater variability in the study sample $(k=0.94, p<0.01$; $k=0.98, p<0.01$; respectively).

Lesion sizes were divided based on measuring of maximum diameter on axial sections by trained neurologists (RRM, HHS) into small $(2-3 \mathrm{~cm})$, medium $(3-5 \mathrm{~cm})$, and large $(>5 \mathrm{~cm})$ infarctions.

\section{Outcome measures}

NIHSS was assessed at baseline, at 1 week (or discharge from hospital) and at 1 month after onset. Modified Rankin Scale (mRS) was assessed at 3 months.

Informed consent was obtained from all subjects or their next of kin. The study was approved by the Ain Shams University Local Research Ethics Committee.

\section{Statistical analysis}

Comparisons were made between groups (homogeneous vs. non-homogenous DWI lesion) in mRS at 3 months using the Mann-Whitney $U$ test (median and interquartile range [IQR]) and in NIHSS at baseline, 1 week and at 1 month using repeated-measures ANOVA. Comparison of lesion sizes between groups was done using chi-squared test. The analysis was done on SPSS ver. 20 (IBM SPSS, $\mathrm{NY}$, USA). $p<0.05$ was considered significant.

\section{Results}

Fifteen patients fulfilling the criteria were recruited with non-homogenous DWI lesions and 15 with homogenous DWI lesions. Of the latter group, 3 patients died in hospital $<1$ week after onset $(\mathrm{mRS}=6)$ and were excluded from further analysis of NIHSS. Patient characteristics

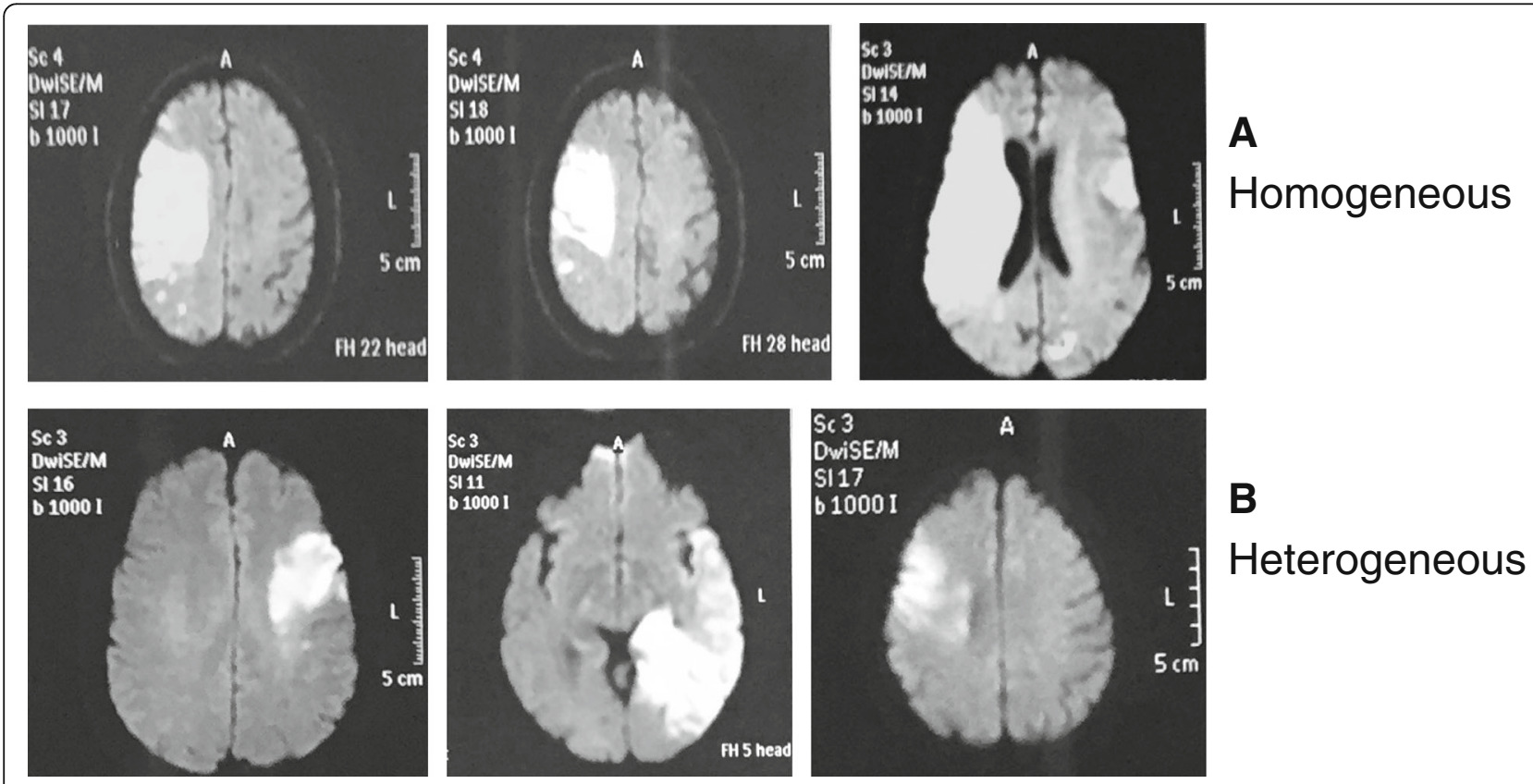

Fig. 1 Examples of homogenous (top row, a) and heterogeneous (bottom row, b) DWI lesions 
Table 1 Patient characteristics

\begin{tabular}{|c|c|c|}
\hline & Homogenous DWI & Non-homogeneous DWI \\
\hline Age & $59.2 \pm 10.0$ & $62.0 \pm 15.6$ \\
\hline Gender & $7 \mathrm{M}, 8 \mathrm{~F}$ & $7 \mathrm{M}, 8 \mathrm{~F}$ \\
\hline Diabetes mellitus & $6 / 15$ & $4 / 15$ \\
\hline Hypertension & $8 / 15$ & $9 / 15$ \\
\hline \multicolumn{3}{|l|}{ NIHSS } \\
\hline Baseline & $12.3 \pm 4.7$ & $9.6 \pm 6.9$ \\
\hline 1 week & $10.7 \pm 5.3$ & $5.7 \pm 4.6^{a}$ \\
\hline 1 month & $8.1 \pm 4.5$ & $2.8 \pm 2.3^{\mathrm{a}}$ \\
\hline \multicolumn{3}{|l|}{ Lesion size } \\
\hline Small & 2 & 3 \\
\hline Medium & 9 & 10 \\
\hline Large & 4 & 2 \\
\hline
\end{tabular}

${ }^{\mathrm{a} D a t a}$ available for 12 patients only

are presented in Table 1. There was no significant difference at baseline between the groups in the distribution of lesion sizes $\left(\chi^{2}=0.9, p=0.6\right)$, stroke risk factors (all $p<0.05)$, and severity of stroke on NIHSS (12.3 \pm 4.7 vs. $9.6 \pm 6.9, p=0.22$ ).

\section{Outcome}

Patients with a homogenous DWI lesion had significantly higher mRS score at 3 months, i.e., worse outcome, than those with a non-homogenous (heterogeneous) DWI lesion (median, IQR $4,2-4$ vs. 1, 0-2 respectively, $p=$ 0.001; Fig. 2a). Similarly, for NIHSS, although there was no difference between the groups at baseline, repeated measures ANOVA showed a significant main effect of time $(F(2,50)=36.0, p<0.001)$ and group $(F(1,25)=5.77$, $p=0.024)$ but not time $\times$ group interaction $(F(2,50)=$ $2.27, p=0.1$, Fig. 2b).

\section{Discussion}

This study describes a novel marker of ischemic stroke prognosis based on visual assessment of routine MR DWI lesions. We demonstrate that patients with anterior circulation non-lacunar infarcts of all sizes who have a homogeneous DWI lesion type have worse outcome on 3 months mRS and 1 month NIHSS than similar patients with non-homogenous (heterogeneous) DWI lesions.

DWI lesion homogeneity is a simple and easy feature that can be accurately determined using visual inspection alone in a single scanning session with no additional scanning time or special analysis techniques. This makes it particularly suited to the clinical situation where a decision to proceed with further treatments or withhold it needs timely information.

The volume of the early DWI lesion has repeatedly been found to be an independent predictor of outcome [3]. A cutoff volume $>70 \mathrm{ml}$ has recently become accepted as a poor prognostic marker and has even been used in thrombectomy trials to exclude patients from therapy [13]. Nonetheless, this has been contested [7, 14], emphasizing that volume alone may not be sufficient to exclude potential patients or predict outcome [15]. Combination with MRA (to assess recanalization) $[2,7]$ and characterization of the DWI lesion itself in terms of pattern [6] and location [4] have been proposed to improve prediction of prognosis.

It is now established that at least at early time points, parts of the DWI may represent viable penumbral tissue that can be salvaged $[11,12]$, and hence not contribute to final infarct volume (partial DWI lesion reversal). It can be proposed that heterogeneity of the DWI may

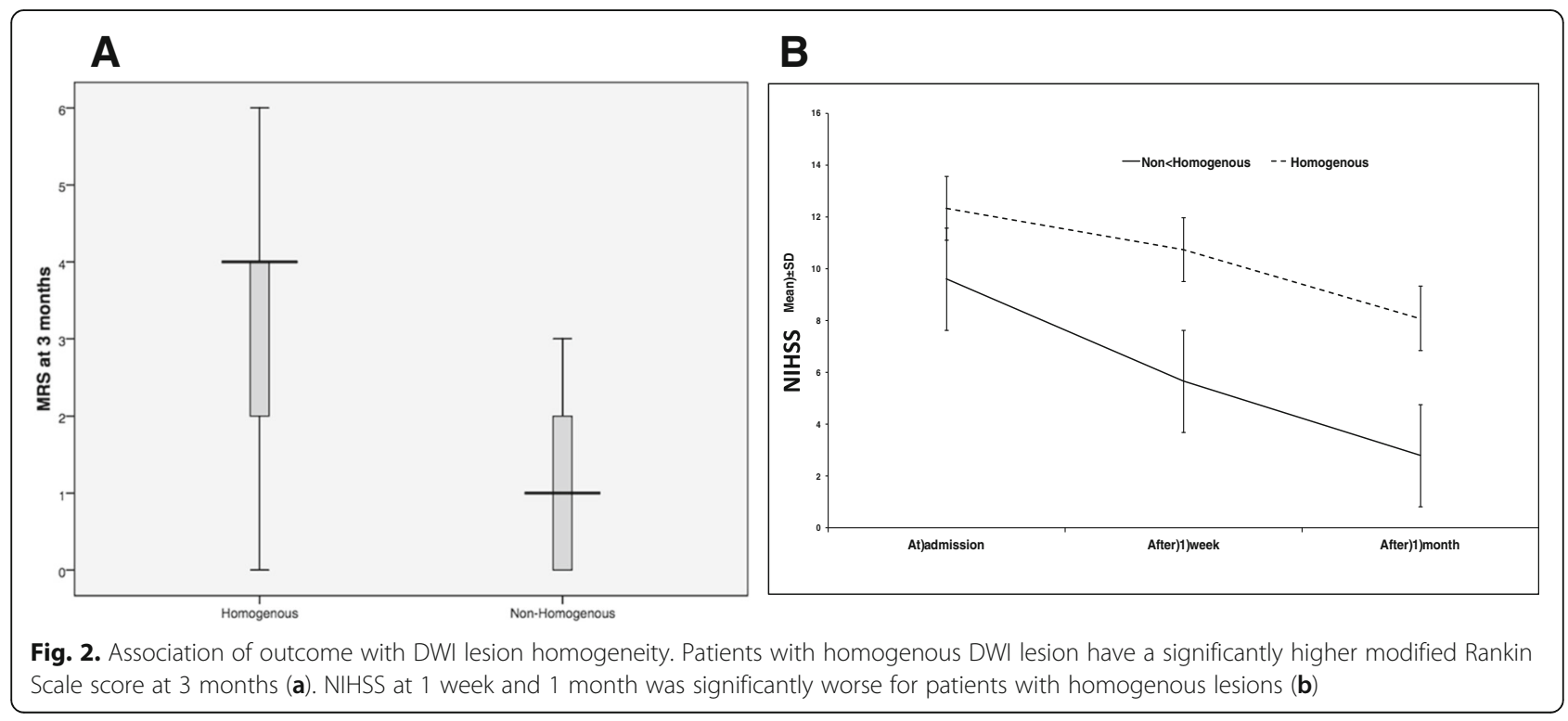


reflect this variable fate of the DWI lesion, whereas homogeneity may indicate inevitable infarction of the entire DWI lesion. Cerebral perfusion studies may clarify if this is the case and a longitudinal MRI study, particularly in patients who achieve recanalization, will be able to establish if this is the reason behind the difference in prognosis between these two types as observed in the present study.

This study is limited by the small number of patients and by not using a quantitative method for calculating lesion volume accurately. Additional confirmation in a larger sample of patients is thus important and should include measurement of other imaging predictors to ascertain their independence of each other and their individual predictive power. Also, exact measurement of $\mathrm{ADC}$ and variance in intensity within the DWI lesion could be useful in future studies to establish the quantitative cut-off points that correspond to the visual assessment of lesion homogeneity.

\section{Conclusion}

Lesion homogeneity on early MR DWI is a strong marker of outcome in acute ischemic stroke. It is a simple and reproducible visual assessment and may be useful in tailoring therapy for the individual patient in the clinical setting.

\section{Abbreviations}

MRI: Magnetic resonance imaging; DWl: Diffusion-weighted imaging; MRS: Modified Rankin Scale; NIHSS: National Institutes of Health Stroke Scale; ANOVA: Analysis of variance; IQR: Interquartile range; ADC: Apparent diffusion coefficient

\section{Acknowledgements}

Not applicable

\begin{abstract}
Authors' contributions
SMB collected the patient data, identified patients, and did the analysis. RRM designed the study, contributed to the data collection, statistical analysis, and manuscript writing. MAM supervised the work and revised the manuscript. AS contributed to the study design and manuscript revision. MMM contributed to study design and revising the results. HMA suggested the study idea, contributed to the study design, and revised the results and manuscript. All authors read and approved the final manuscript.
\end{abstract}

\section{Funding}

Not applicable

\section{Availability of data and materials}

Dataset is available as a master sheet in Excel format and publicly available in Neurology Department, Ain Shams University, through communicating with the corresponding authors

\section{Ethics approval and consent to participate}

The study was approved by Ain Shams University Ethical Committee in 2016. Written informed consent was obtained from the patients participating in the study.

\section{Consent for publication}

Not applicable

\section{Competing interests}

The authors declare that they have no competing interests.
Received: 25 May 2018 Accepted: 5 August 2019

Published online: 03 September 2019

\section{References}

1. Heiss W-D, Kidwell CS. Imaging for prediction of functional outcome and assessment of recovery in ischemic stroke. Stroke. 2014;45:1195-201.

2. Nighoghossian N, Hermier M, Adeleine P, Derex L, Dugor JF, Philippeau F, et al. Baseline magnetic resonance imaging parameters and stroke outcome in patients treated by intravenous tissue plasminogen activator. Stroke. 2003; 34:458-63.

3. Thijs VN, Lansberg MG, Beaulieu C, Marks MP, Moseley ME, Albers GW. Is early ischemic lesion volume on diffusion-weighted imaging an independent predictor of stroke outcome? A multivariable analysis. Stroke. 2000;31:2597-602

4. Yassi N, Churilov L, Campbell BCV, Sharma G, Bammer R, Desmond PM, et al. The association between lesion location and functional outcome after ischemic stroke. Int. J. Stroke. 2015;10:1270-6.

5. Kimura K, Iguchi Y, Shibazaki K, Terasawa Y, Inoue T, Uemura J, et al. Large ischemic lesions on diffusion-weighted imaging done before intravenous tissue plasminogen activator thrombolysis predicts a poor outcome in patients with acute stroke. Stroke. 2008;39:2388-91.

6. Bang OY, Lee PH, Heo KG, Joo US, Yoon SR, Kim SY. Specific DWI lesion patterns predict prognosis after acute ischaemic stroke within the MCA territory. J. Neurol. Neurosurg. Psychiatry. 2005;76:1222-8.

7. Tisserand M, Turc G, Charron S, Legrand L, Edjlali M, Seners P, et al. Does diffusion lesion volume above $70 \mathrm{~mL}$ preclude favorable outcome despite post-thrombolysis recanalization? Stroke. 2016;47:1005-11.

8. Albers GW. Diffusion-weighted MRI for evaluation of acute stroke. Neurology. 1998;51:S47-9.

9. Roberts TPL. Rowley H a. Diffusion weighted magnetic resonance imaging in stroke. Eur. J. Radiol. 2003:45:185-94.

10. Kranz PG, Eastwood JD. Does diffusion-weighted imaging represent the ischemic core? An Evidence-Based Systematic Review. Am. J. Neuroradiol. 2009;30:1206-12.

11. Guadagno JV, Warburton EA, Jones PS, Fryer TD, Day DJ, Gillard JH, et al. The diffusion-weighted lesion in acute stroke: heterogeneous patterns of flow/metabolism uncoupling as assessed by quantitative positron emission tomography. Cerebrovasc. Dis. 2005;19:239-46.

12. Alawneh JA, Moustafa RR, Marrapu ST, Jensen-Kondering U, Morris RS, Jones PS, et al. Diffusion and perfusion correlates of the 18F-MISO PET lesion in acute stroke: pilot study. Eur. J. Nucl. Med. Mol. Imaging. 2014;41:736-44.

13. Campbell BCV, Mitchell PJ, Kleinig TJ, Dewey HM, Churilov L, Yassi N, et al. Endovascular therapy for ischemic stroke with perfusion-imaging selection. N. Engl. J. Med. 2015;372:1009-18.

14. Gilgen MD, Klimek D, Liesirova KT, Meisterernst J, Klinger-Gratz PP, Schroth $\mathrm{G}$, et al. Younger stroke patients with large pretreatment diffusion-weighted imaging lesions may benefit from endovascular treatment. Stroke. 2015;46: 2510-6.

15. Hand PJ, Wardlaw JM, Rivers CS, Armitage PA, Bastin ME, Lindley Rl, et al. MR diffusion-weighted imaging and outcome prediction after ischemic stroke. Neurology. 2006;66:1159-63.

\section{Publisher's Note}

Springer Nature remains neutral with regard to jurisdictional claims in published maps and institutional affiliations. 